UCRL-JC-117554

PREPRINT

\title{
Processing and Characterization of High Porosity Aerogel Films
}

\author{
Lawrence W. Hrubesh \\ John F. Poco
}

This paper was prepared for submittal to the

Materials Research Society Fall 1994 Meeting

Boston, MA

November 28-December 2, 1994

November 22, 1994

This is a preprint of a paper intended for publication in a journalor proceedings. Since changes may be made before publication, this preprint is made available with the understanding that it will not be cited or reproduced without the permission of the author.

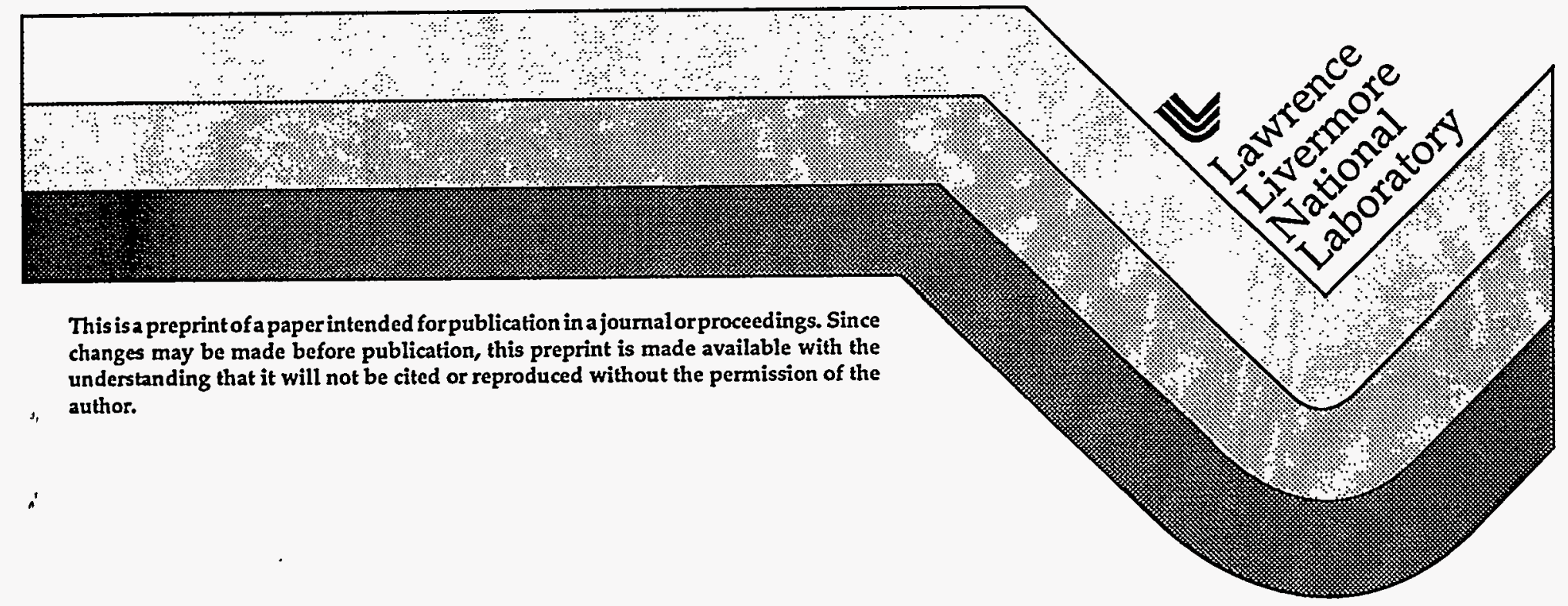




\section{DISCLAIMER}

This document was prepared as an account of work sponsored by an agency of the United States Government. Neither the United States Government nor the. University of California nor any of their employees, makes any warranty, express or implied, or assumes any legal liability or responsibility for the accuracy, completeness, or usefulness of any information, apparatus, product, or process disclosed, or represents that its use would not infringe privately owned rights. Reference herein to any specific commercial product, process, or service by trade name, trademark, manufacturer, or otherwise, does not necessarily constitute or imply its endorsement, recommendation, or favoring by the United States Government or the University of California. The views and opinions of authors expressed herein do not necessarily state or reflect those of the United States Government or the University of California, and shall not be used for advertising or product endorsement purposes. 


\title{
PROCESSING AND CHARACTERIZATION OF HIGH POROSITY AEROGEL FILMS*
}

\author{
LAWRENCE W. HRUBESH AND JOHN F. POCO \\ Chemistry and Material Science Department \\ Lawrence Livermore National Laboratory \\ Livermore, California 94550
}

\begin{abstract}
Aerogels are highly porous solids having unique morphology among materials because both the pores and particles making up the material have sizes less than wavelengths of visible light. Such a unique morphology modifies the normal molecular transport mechanisms within the material, resulting in exceptional thermal, acoustical, mechanical, and electrical properties. For example, aerogels have the lowest measured thermal conductivity and dielectric constant for any solid material. Special methods are required to make aerogel films with high porosity. In this paper, we discuss the special conditions needed to fabricate aerogel films having porosities greater than $75 \%$ and we describe methods of processing inorganic aerogel films having controllable thicknesses in the range 0.5 to 200 micrometers. We report methods and results of characterizing the films including thickness, refractive index, density (porosity), and dielectric constant. We also discuss results of metallization and patterning on the aerogel films for applications involving microminiature electronics and thermal detectors.
\end{abstract}

*Work performed under the auspices of the U.S. Department of Energy by the Lawrence Livermore National Laboratory under Contract No. W-7405-ENG-48. 


\section{INTRODUCTION}

Aerogels are very high porosity (typically $>75 \%$ ) materials made by sol-gel chemistry that forms solvent filled gels which are dried using special conditions to preserve the tenuous solid network [1,2]. The dried aerogel is an air-filled porous solid, usually in monolithic or granular form. The microstructure of aerogels consists of particles and pores which are only fractions of the wavelength of visible light in size. Such structure is unique among common materials and many extraordinary properties result from it. For example, aerogels are known to exhibit the lowest thermal conductivity, sound velocity, and refractive index, of any bulk solid material [3]. The microstructure and the very high porosity of aerogels are also responsible for the exceptional optical, thermal, acoustical, and electronic properties of aerogels. Most of the properties which are characteristic for bulk aerogels are also exhibited in other forms of the material, e.g., in thin sheets or films. A growing number of electronic and thermal applications require the aerogels as thin layers or films on other substrate materials. Fabricating the aerogels in such forms presents problems due to the weak, tenuous nature of the highly porous aerogels and because special requirements are necessary to form and dry them.

Sol-gel processes have been well developed as a means for producing thin films of advanced glasses, glass-ceramics, and ceramics for a variety of applications. There are several books and review articles on sol-gel produced films and coatings, which describe the advantages and details of sol-gel films for optical, electronic, and other applications [4-8]. The formation of thin aerogel films might seem rather straightforward because of the considerable success in applying sol-gel coatings to surfaces, however, the rapid evaporation of solvent that accompanies the deposition processes causes the sols to form compact films as drying occurs, rather than forming a more porous gel. Therefore, special considerations and methods are needed to successfully form highly porous gels and dry them to make low density aerogels. Very little work has been reported for processing porous sol-gel films where the films are to remain porous for their application. There are some articles which discuss sol-gel films having porosities as high as . 50\% [9-11] and one article discusses the preparation of an aerogel film but the porosity is not reperted [12]. Here we describe the special conditions and requirements which are needed to make aerogel films having porosities $>75 \%$ and we discuss several applications for such films.

\section{EXPERIMENTAL PROCEDURES}

Most of the methods already developed for applying sol-gel thin films and coatings (e.g., dipping, spinning, spraying, etc.) generally apply to making true aerogel films. However, all of these methods have the common requirement that the gel must be formed under conditions in which the rate of evaporation is limited, both during and after the gel formation. To facilitate this we perform the coating processes within an enclosure that is maintained saturated with the vapor of the working solvent. The atmosphere in the enclosure also contains a partial pressure of ammonium hydroxide which catalyzes the gelation of the films. The most common aerogel films are silica, but aerogel films have also been demonstrated with other metal oxides for which hydrolysis/condensation of the metal alkoxide is the predominant chemistry (e.g., zirconia,

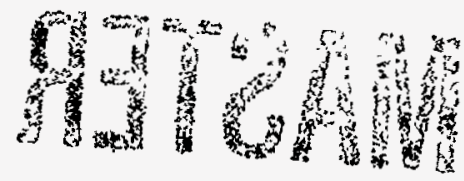


alumina, and tantala). We used two methods to prepare the precursor solution for the process depending on the desired porosity of the final aerogel. For gel porosities $>95 \%$ we used the twostep, partial hydrolysis/condensation chemistry reported previously for silica aerogels [13]. For gels with lower porosities, we prepared a single-step, base-catalysed hydrolysis solution according to the common method for silica as.follows: mix tetramethoxysilane (TMOS), water, methyl alcohol, and ammonium hydroxide, in a molar ratio of 1:2:4:0.01. An additional amount of alcohol is added to this mixture to establish the final porosity of the gel. The methods used to make thin aerogel films depend on the thickness desired and whether or not the film is to be bonded to a surface.

The flow chart in figure 1 shows the processing steps leading to high porosity aerogel films. In the following we discuss the various processing steps and procedures to form thin aerogel films on various substrate materials.

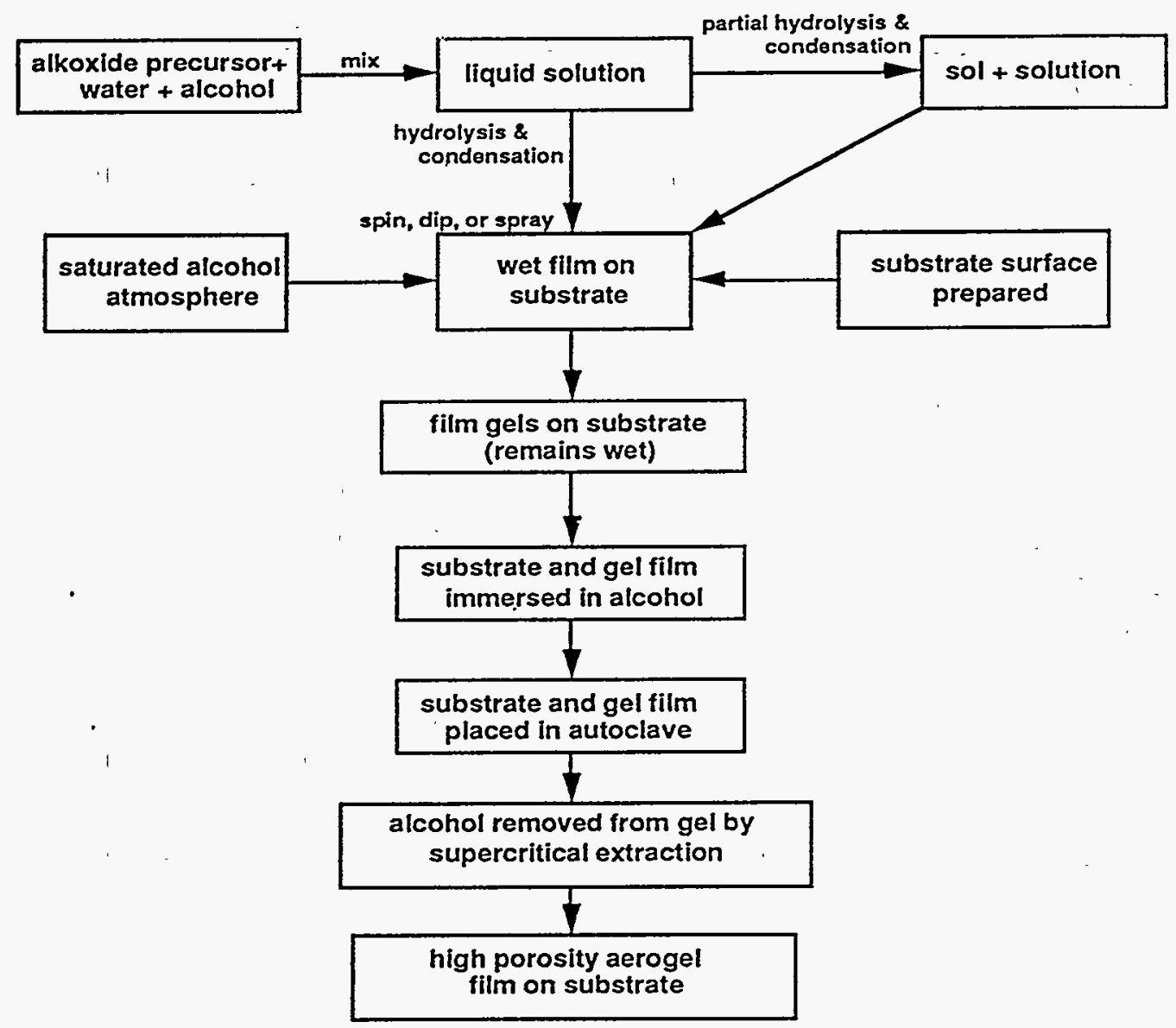

Figure 1. Flow chart for preparation of high porosity aerogel films

\section{Surface Preparation}

The preparation of the substrate surface is critical for good quality aerogel films. We have found that most of the metal oxide gels adhere to glass or oxidized surfaces (probably through 
metal-oxide-metal bonds) but the gels do not stick well to unprepared metal surfaces. For metal surfaces such as silicon wafers, a $100 \mathrm{~nm}$ thick silicon dioxide coating on the surface is sufficient to cause bonding of the aerogel to the surface. Bonding to all oxidized surfaces is enhanced by hydroxylating the surface using a mild alkaline solution (e.g, $\mathrm{KOH})$, then rinsing with alcohol immediately prior to film deposition. When non-bonding was desired, the oxidized surfaces were treated with a methylated silicate compound that served as a release agent.

\section{Spin, Dip, and Spray Coatings}

Spin coating for aerogels is essentially the same as that used to spin glass coatings for electronic applications, except that it is performed with the spin apparatus entirely within an enclosure that has a solvent saturated atmosphere. Film thicknesses are typically less than two micrometers. Special modifications were performed on the apparatus to prevent the possibility of an explosive hazard. Typical substrates are glass slides and silicon wafers up to 3" diameter. The procedure for forming films is to meter a droplet of precursor solution onto the spinning substrate while its spin rate is increasing up to a maximum speed of 1650 RPM. The spinner is immediately turned off and stopped with a brake, so that the sample is subject to minimal loss of solvent during gelation. Typically, the gel will form within a few minutes, after which the substrate is manually removed from the spin apparatus and immersed in solvent. The substrates with films are stored submersed in solvent until ready for supercritical drying.

Dip coating is used when all surfaces of a substrate material are to be coated. With this method, film thicknesses less than a few micrometers are obtainable depending on the viscosity of the precursor and the withdrawl rate. In our work, the precise thickness was not an important parameter, so the dipping and withdrawing procedure is performed manually without concern for controlling the rates. Typical substrates are glass slides of various sizes. The sustrates are simply dipped into the prepared precursor solution, withdrawn, then placed edgewise and vertical in a holder which is located within the enclosure. The time for gel formation is typically only a few minutes. After the film is gelled, the entire holder containing the slides is immersed in a beaker of solvent and it remains surrounded by liquid until ready for supercritical drying.

Spray coating has been used to put thicker single layer coatings on substrates like glass and silicon wafers. Films as thick as 80 micrometers have been acheived by this method. An aspirator is used to spray the precursor solution directly onto the substrate which is supported in a nearly vertical position within the enclosure. Excess solution drains by gravity, leaving a thick film which gels within a few minutes. These films have a varying thickness due to the draining, but the surface of the gel is smooth and continuous. After gelation, the substrate is immersed in solvent until ready for supercritical drying.

\section{Supercritical Drying}

In order to prevent densification of the gel films and maintain high porosity aerogels, supercritical drying methods were used to dry the solvent filled gel films. Either direct supercritical extraction (SCE) of the solvent was done at high temperature or an alternate low temperature extraction of carbon dioxide was performed after exchange of the original solvent. 
The chosen method of drying depends on the temperature stability of the substrate material. Glass substrates and silicon wafers with a protective coating of silicon dioxide are generally compatible with high temperature SCE whereas plastics and some uncoated metals will not survive the high temperature SCE of solvents. The procedure for direct SCE is to place the glass container holding the submersed (or encapsulated) samples directly into an autoclave. The autoclave is filled with additional solvent (usually, alcohol) and sealed. The temperature of the autoclave is raised at a rate of $0.3^{\circ} \mathrm{C} / \mathrm{min}$ while the pressure increases to about 120 bars and excess pressure above that is released. After the temperature reaches about $280^{\circ} \mathrm{C}$, the pressure is released from the vessel at a controlled rate of $0.3 \mathrm{bar} / \mathrm{min}$ until a pressure of about $1.5 \mathrm{bar}$ is reached. The autoclave is then purged with air as the vessel cools. This cycle typically takes 20 hours. Alternatively, a similar cycle is performed at temperatures less than $45^{\circ} \mathrm{C}$ after the solvent in the gel-film is first exchanged with liquid carbon dioxide. The exchange time to remove solvent from the thin films which are less than 50 micrometers thick is typically 2 hours, if the entire surface of the gel-film is exposed within the autoclave.

\section{Metallization and Patterning}

Metals can be deposited directly on as-prepared aerogel surfaces by vapor or sputter deposition processes. The brief intense heating of the aerogel surface in these methods does not seem to adversely affect the quality of the metal coatings. Metals including $\mathrm{Au}, \mathrm{Pt}, \mathrm{Cu}, \mathrm{Al}, \mathrm{Cr}$, and $\mathrm{Ti}$, have been directly deposited onto silica aerogel films, and metal coating thicknesses from a few nanometers up to 5 micrometers have been successfully achieved. For some cases, low resolution patterning is achieved by depositing the metal through an appropriate mask. Also, higher resolution metal patterning may be accomplished by photoresist methods after sealing the aerogel surfaces. For example, approximately $1000 \AA$ of polymer (e.g., parylene and trans-2butene) is sufficient to seal the pores of the aerogel. Photoresist is spun on over the sealed surface and processed to form patterns. The sealing polymer coating can be etched away (dry etch) after metallization patterning is completed.

\section{FILM CHARACTERIZATION}

The morphology of the films and the film-substrate interface regions may be examined using a high resolution scanning electron microscope (SEM). The film thicknesses are measured using a stylus type profilometer. Film adherence on glass and silicon wafers are qualitatively determined using a tape stick test with Scotch ${ }^{\otimes}$ tape.

Refractive indexes of films were measured using an ellipseometer. This instrument will measure either the refractive index or the thickness of a thin dielectric layer if the other is known. The thickness measured by a profilometer is entered as input to the ellipsometer to calculate the refractive index of a film. Generally, it is difficult to apply this technique with aerogels because the surface reflectivity is typically less than $1 \%$ at a wavelength of $632.8 \mathrm{~nm}$. However, sufficient reflectivity is usually obtained at shallow angles of incidence. 
Once successful measurements of the refractive indexes are made, other physical properties of the aerogel films are determined as follows: The film densities are calculated using the measured value of the optical refractive index, $n$, by applying the Dale-Gladstone relation, $\rho$ $=(n-1) / 209$, reported to hold for silica aerogels [14]. The dielectric constant and the porosity of the films are also determined from the density, after measurement of the optical refractive index. The porosity is determined from the relation, $\Pi=1-\rho / \rho s$, where $\rho s$ is the density of the solid. For example, for silica aerogel with $\rho s=2.19 \mathrm{~g} / \mathrm{cc}, \Pi=[(1.458-\mathrm{n}) / 0.458] \times 100$ is the percent porosity. About $1 \%$ lower values are obtained using a similar relation for the porosity as a function of refractive index derived from the Lorentz-Lorenz equation [15], $\Pi=1-\left\{3.66\left[\left(\mathrm{n}^{2}-\right.\right.\right.$ 1)/(n $\left.\left.\left.n^{2}+2\right)\right]\right\}$. The dielectric constants for the silica aerogel films or sheets are determined after measurement of the optical refractive index using: $\kappa^{\prime}=1+1.6 p[16]$, or $\kappa^{\prime}=1+7.7(n-1)$. Figure 2 shows how the refractive index and dielectric constant change as a function of porosity for silica aerogels having porosities $>70 \%$.

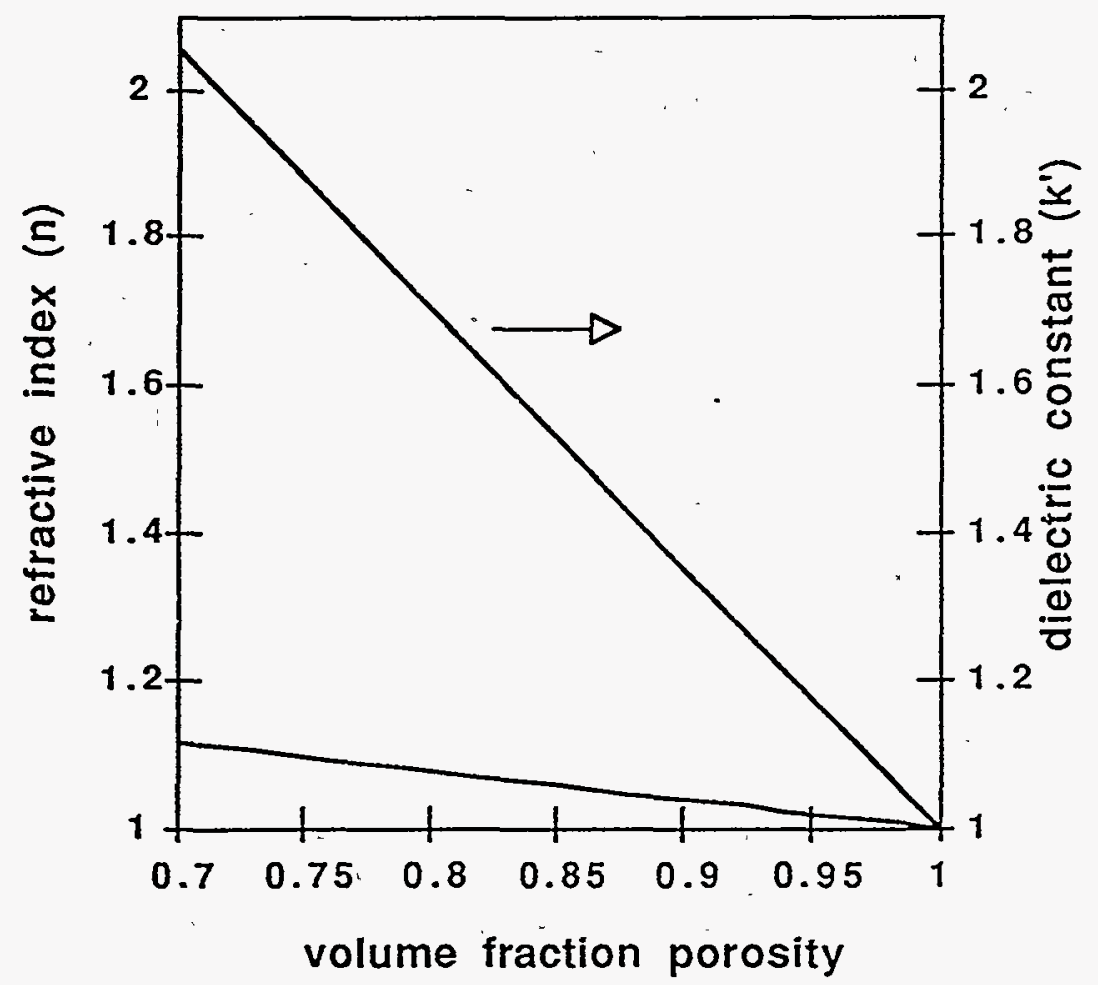

Figure 2. Refractive index and dielectric constant of silica aerogels as a function of porosity.

The thermal conductivity of thin aerogel films is usually inferred from measurements made on the bulk materials. Direct measurement of thermal conductivity of the films is difficult and methods for such measurements are under currently development. 


\section{RESULTS}

Using the methods described, we have successfully fabricated thin, flat, uniform silica aerogel films, having various porosities, with measured thicknesses from less than 1 to 20 micrometers on glass and silicon wafer substrates. The adherence of the films to the substrates has been qualitatively determined by a tape stick test. Generally, good film adherence was obtained for all aerogels with a porosity less than about $86 \%$ and mixed results were observed for porosities between $86 \%$ and $95 \%$. For porosities $>95 \%$, the results were invalid because the aerogel is too weak to survive the application of the tape. These films have been examined using SEM to verify their aerogel-like structure.

The densities and porosities which were calculated from the refractive index measurements for these films, confirms that they are aerogels having densities in the range from about $0.04-0.46 \mathrm{~g} / \mathrm{cc}$, and porosities in the range from $77 \%$ to $98 \%$. We have also calculated the dielectric constants of these films based on the measured refractive indexes. The dielectric constants span the range from about 1.06 to 1.7. Table I shows characteristic data for some representative aerogel films.

Table I. Characteristic data for some representative thin aerogel films on glass

\begin{tabular}{lccccc}
\hline Film type & Method & $\begin{array}{c}\text { Ave. thickness }(\mu \mathrm{m}) \\
\text { (measured) }\end{array}$ & $\begin{array}{c}\text { Refractive index } \\
\text { (measured) }\end{array}$ & $\begin{array}{c}\text { Porosity (\%) } \\
\text { (calculated) }\end{array}$ & $\begin{array}{c}\text { Diel. constant } \\
\text { (calculated) }\end{array}$ \\
\hline Silica & capillary & 18.1 & 1.062 & 86 & 1.48 \\
Silica & spin & 3.4 & 1.103 & 78 & 1.79 \\
Silica & spray & 44.1 & 1.049 & 89 & 1.38 \\
Zirconia & capillary & 24.7 & 1.059 & 95 &.$--{ }^{--}$ \\
Silica & capillary & 113.5 & 1.009 & 98 & 1.07 \\
\hline
\end{tabular}

Processes to seal, pattern, and metallize aerogel films have been developed. An example of a high resolution pattern of gold metal on an aerogel film is shown in figure 3 . This pattern of 2 micrometer wide strips was achieved with the following steps: 1) fabricate a 3.4 micrometer thick silica aerogel film on a $7.6 \mathrm{~cm}$ diameter silicon wafer using spinning and supercritical drying, 2) vapor deposit $\sim 100 \mathrm{~nm}$ parylene onto aerogel film, 3) vapor deposit $40 \mathrm{~nm}$ titanium metal then $500 \mathrm{~nm}$ gold, 4) spin on $1500 \mathrm{~nm}$ photoresist and dry at $\left.90^{\circ} \mathrm{C}, 5\right)$ mask and expose to ultraviolet, 6) remove mask, wash with $\mathrm{KOH}, 7$ ) etch away gold, 8) plasma etch titanium down to parylene, 9) ash away the remaining resist, leaving a metallized pattern on parylene coated aerogel.

We have also sputtered thin $(<0.5$ micrometer) metal layers directly onto the aerogel surfaces and electroplated thicker ( $>1.0$ micrometer) layers on the sputtered layers. 


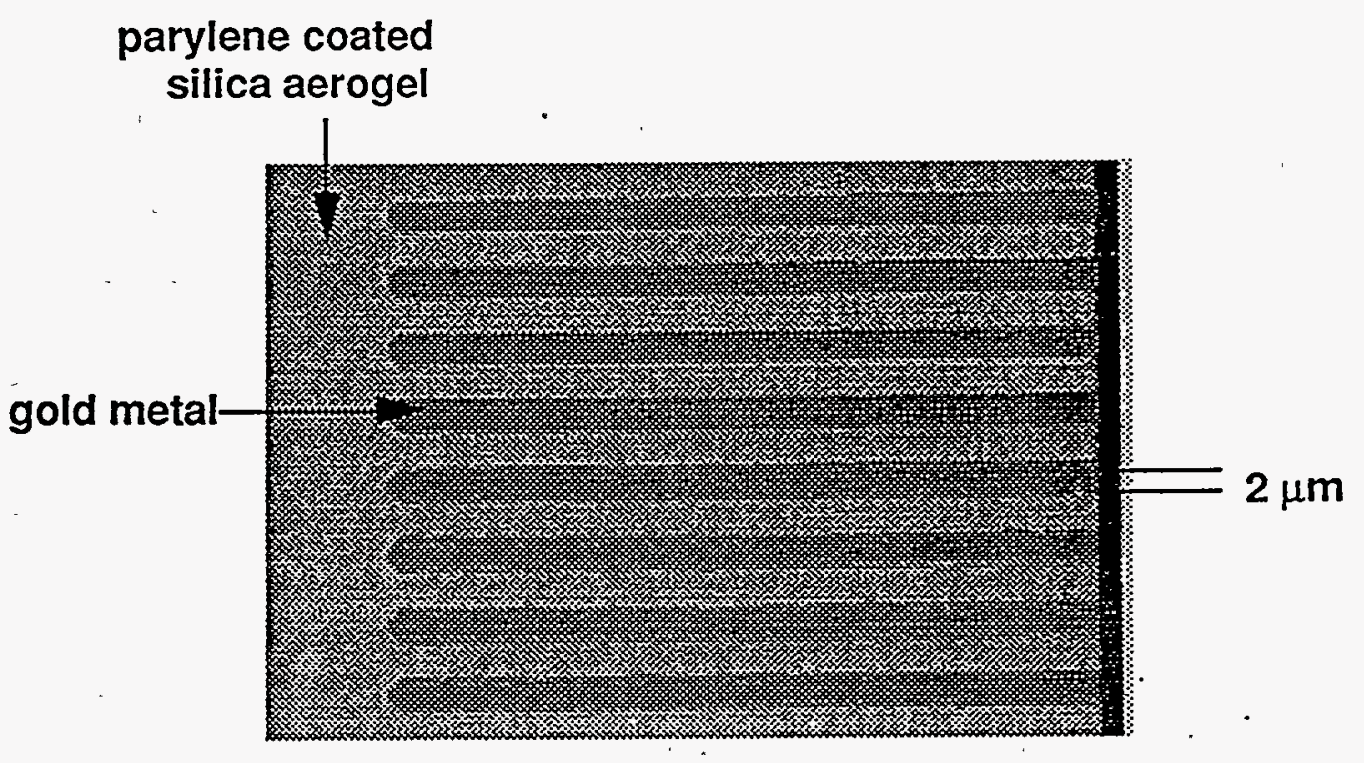

Figure 3. Gold metal patterned on silica aerogel

\section{APPLICATIONS OF AEROGEL FILMS}

Aerogel films can serve in many useful applications. Here we describe several specific applications for which we have processed thin aerogel films. In most cases, the aerogel films are used on prototypes which are currently being tested for functionality and performance.

\section{Optical .}

Thick aerogel films ( $\approx 0.2$ millimeter) have been fabricated for use as coverslips on solar cells. In this case the aerogel films are formed, then transferred to the solar cell surface and bonded to it, instead of being formed directly on it. By replacing the high index glass coverslips normally used, the low refractive index aerogel causes less reflective Fresnel loss for incident light at its outer surface, allowing more light to reach the active surface, thus increasing the solar cell efficiency.

Thin aerogel films ( $\approx 30$ micrometers) have been deposited as a cladding on the outside of glass laser pump tubes. In this application, the aerogel acts as a refractive index match between the pump tube glass and air, to minimize reflection of internally generated light at the outer envelop of the tube, thus increasing the amount of excitation light reaching the lasing medium. The aerogel is preferred to antireflection coatings in this application because it provides a better match for a broader band of light waves. 


\section{Thermal}

Thin aerogel films $(\approx 25$ micrometers) have been formed on glass substrates for use in "cool" IR detectors. Here, the aerogel film serves as a thermal barrier to shield the IR detector elements from the heat radiated by the substrate materials.

Many thick sheets ( $\approx 1$ millimeter) of aerogel are coated with thin layers of metal, then laminated together to form a super-insulating thermal heat shield block.

\section{Acoustic}

Thick aerogel films $(=0.5$ millimeter) are formed on the suface of ceramic transducers to serve as acoustic impedance matching layers. The efficiency of sending and receiving airborne acoustic waves is substantially increased by using aerogels in this application.

\section{Electronic}

Thin aerogel films $(\approx 2$ micrometers) are formed on silicon wafers to provide a low dielectric constant substrate in integrated circuits. The dielectric constant for all aerogels is less than 2.0 and its value depends on the porosity, a controllable parameter for aerogel films. Dielectric constants of the aerogels well below 2.0 will enable significant improvements in the speed of integrated circuits.

Several thick organic aerogel films $(\approx 0.5$ millimeter) are formed by capillary fill, then pyrolyzed to carbon aerogel films for use in aerocapacitors. These devices can provide specific capacitances in excess of 45 Farads per gram of material [17].

\section{Other applications}

Applications for the thin film dielectrics include: microwave striplines; microwave circuits such as used in radars and communications; low capacitance chip connectors; extremely lightweight electronic packaging; power transmission high voltage insulators; andspacers for electrodes in vacuum tubes.

Some other applications of thin aerogel films include: membranes for gas separations; flat panel displays; window glazing; electrical standoffs; light bulbs for diffusing light; passivation layer for oxidative metals; insulative layer between stacks of metals or polymers; antireflective films; cladding on optical fibers; and optical, chemical sensors.

This is only a partial listing of potential applications for aerogel films. High porosity films appear to have as many applications as the bulk aerogels do. However in both cases, continued development is needed to refine the processes, to reduce processing costs, and to demonstrate the practical uses of the technology. In the case of thin aerogel films, the effort has just begun. 


\section{CONCLUSIONS}

It is possible and practical to form highly porous, true aerogel films on substrates using a variety of common deposition methods, if care is taken to slow evaporation of solvents during gelation, and if supercritical drying is done to preserve their tenuous structure. The aerogel films are good quality, bond well to the substrates, and are strong enough to survive other processing steps used to prepare them for specific applications. Most importantly, the aerogel films exhibit many of the exceptional properties which are characteristic of the bulk material and therefore they can be considered for use in a variety of optical, acoustical, thermal, and electronic applications. We anticipate that with further development, aerogel films will be be used in such applications in the near future.

\section{References:}

1) R.P. Iler, The Chemistry of Silica, (J. Wiley and Sons, New York, 1979) pp.537-539.

2) J.'Fricke, Sci. Am. 256, 92 (1988).

3) J. Fricke and A. Emmerling, in Chemistry, Spectroscopy, and Applications of Sol-Gel Glasses, edited by R. Reisfeld and C.K. Jorgensen, (Springer Series on Structure and Bonding, Vol. 77, Springer-Verlag, Heidelberg, Germany, 1991) p.37.

4) L.C. Klein, Ed.,Sol-gel Technology for Thin Films, Fibers, Preforms, Electronics and Specialty Shapes (Noyes, Park Ridge, N.J., 1988).

5) D.R. Ulrich, J. Non-Cryst. Solids 100, 174 (1988).

6) H. Dislich, in Sol-gel Technology for Thin Films, Fibers, Preforms, Electronics and Specialty Shapes, edited by L.C. Klein (Noyes, Park Ridge, N.J., 1988) p. 50.

7) C.J. Brinker and G.W. Sherer, Sol-Gel Science (Academic Press, N.Y., 1990).

8) C.J. Brinker, A.J. Hurd, P.R. Shrunk, G.C. Frye and C.S. Ashley, J. Non-Cryst. Solids 147, 424 (1992).

9) C.J. Brinker, A.J. Hurd, K.J. Ward, in Ultrastructure Processing of Advanced Ceramics, edited by J.D. Mackenzie and D.R. Ulrich (Wiley, New York, 1988) p. 223.

10) H. Schmidt, G. Rinn, R. Nass and D. Sporn, in Bett. Ceram. for Chem. III, edited by C.J. Brinker, D.E. Clark and D.R. Ulrich (Mat. Res. Soc., Pittsburgh, 1988) p.743.

11) H. Hirashima and K. Sudoh, J. Non-Cryst. Solids 145, 51 (1992).

12) R. Sempere', D. Bourret, A. Sivade, R. Etienne and J. Bouaziz, J. Non-Cryst. Solids 148, 499 (1992).

13) L.W. Hrubesh, T.M. Tillotson, and J.F. Poco, in Chemical Processing of Advanced Materials, edited by L.L. Hench and J.K. West (Wiley, New York, 1992) p. 19.

14) S. Henning and L. Svensson, Phys. Scripta 23, 697 (1981).

15) M. Born and E. Wolf, in Principles of Optics (Pergamon, New York, 1975) p.87.

16) L.W. Hrubesh, L.E. Keene, and V.R. Latorre, J. Mater. Res. 8, 1736 (1993).

17) S.T. Mayer, R.W. Pekala and J.L. Kaschmitter, J. Electrochem. Soc. 140, 446 (1993). 


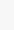


Technical Information Department • Lawrence Livermore National Laboratory University of California • Livermore, California 94551

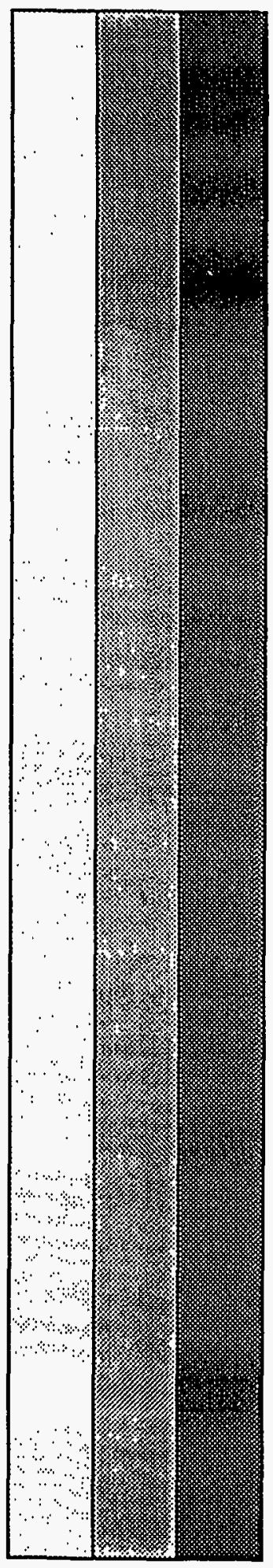

